\title{
De quelques effets pervers des telecommunications en entreprise
}

Francis Jauréguiberry

\section{OpenEdition}

1 Journals

Édition électronique

URL : http://journals.openedition.org/communicationorganisation/1708

DOI : 10.4000/communicationorganisation. 1708

ISSN : 1775-3546

Éditeur

Presses universitaires de Bordeaux

Édition imprimée

Date de publication : 1 mai 1994

ISSN : 1168-5549

Référence électronique

Francis Jauréguiberry, " De quelques effets pervers des telecommunications en entreprise », Communication et organisation [En ligne], 5 | 1994, mis en ligne le 26 mars 2012, consulté le 10 décembre 2020. URL : http://journals.openedition.org/communicationorganisation/1708 ; DOI : https://doi.org/10.4000/communicationorganisation.1708

Ce document a été généré automatiquement le 10 décembre 2020.

(c) Presses universitaires de Bordeaux 


\title{
De quelques effets pervers des telecommunications en entreprise
}

\author{
Francis Jauréguiberry
}

\section{L'entreprise n'est plus concevable sans télécommunications}

1 Le poids des télécommunications dans l'organisation et le fonctionnement des entreprises devient chaque jour plus important. Des secteurs entiers de l'activité économique en sont d'ores et déjà totalement dépendants: les transports, les médias ou la bourse par exemple. Pendant longtemps, l'usage intensif des télécommunications est demeuré l'apanage des entreprises gestionnaires de l'urgence, les autres se contentant d'adopter progressivement le téléphone puis l'informatique. Mais le fantastique développement, ces dix dernières années, des outils de télécommunication (radiotéléphone mobile, télécopie, alphapage et $E D I$ ) a bouleversé ce paysage en permettant à n'importe quelle entreprise, quelle que soit sa taille et son secteur d'activité, d'être médiatiquement proche du géographiquement lointain pour un faible investissement financier. En l'espace d'une décennie, les télécommunications sont devenues indispensables aux entreprises performantes pour au moins trois raisons.

2 La première est celle de l'adaptabilité au marché. L'avant-goût d'ubiquité que procurent les nouveaux outils de télécommunication à l'entreprise moderne lui est devenu indispensable si elle ne veut pas sombrer. Car telle est désormais la loi du genre : dans un univers hyper concurrentiel, l'immédiateté des réponses aux sollicitations du marché devient une règle de survie. Les télécommunications sont ici incontournables: toutes choses étant égales par ailleurs, c'est celui qui aura le plus d'agilité informationnelle et de rapidité d'intervention qui l'emportera.

3 La seconde est liée à l'optimisation des fonctions. Leur rationalisation, mobilisée en vue d'une meilleure efficacité, apparaît de plus en plus nettement liée à la bonne gestion du temps. Une plus grande vitesse de réaction est requise face tant aux aléas internes pouvant affecter la fonction elle-même qu'aux sollicitations externes de plus en plus 
nombreuses et instables dont dépend la fonction. Or les télécommunications sont synonymes de raccourcissement des délais d'information et de réponse. Ainsi, on peut observer que la généralisation de la gestion en juste à temps ou de l'organisation de la distribution en flux tendu se double systématiquement de la mise en place de réseaux et terminaux de télécommunication.

Enfin, la troisième raison renvoie à l'organisation du système de l'entreprise. La dispersion des centres de production, les délocalisations, la nécessité d'être présent sur des marchés éloignés se traduisent par un éclatement géographique chaque fois plus manifeste des fonctions de l'entreprise. La polarisation de l'entreprise localisée cède le pas à l'extension de l'entreprise-réseau. La généralisation de la sous-traitance de certaines tâches jusqu'alors effectuées au sein de l'entreprise, ainsi que la nécessaire fidélisation de la clientèle, étend même cette notion de réseau au-delà des frontières de l'entreprise. Plus dispersée dans un environnement plus fluctuant, l'entreprise se doit d'être plus efficace dans la coordination et la mobilisation de ses différents acteurs. Les télécommunications jouent ici un rôle central en permettant de gérer en temps réel l'organisation inter-fonctionnelle de l'entreprise.

5 Adaptabilité au marché, optimisation des fonctions et organisation du système de l'entreprise-réseau: à chaque fois les télécommunications deviennent indispensables. Mais les fantastiques économies de temps et de déplacements qu'elles permettent ne doivent pas faire oublier qu'elles peuvent engendrer des bouleversements dans les rapports humains. Sous couvert de célébration technologique, beaucoup pensent que ces bouleversements sont nécessairement positifs. L'observation des acteurs de l'entreprise télécommunicante donne au contraire à penser que ces bouleversements peuvent aussi être négatifs en ce qu'ils sont en train de produire un certain nombre d'effets pervers. Si l'on n'y prend pas garde, ces effets risquent d'engendrer de nouveaux dysfonctionnements à défaut de susciter de nouveaux rapports conflictuels autour d'enjeux inédits liés aux usages des télécommunications en entreprise ${ }^{1}$.

\section{Du juste à temps au toujours-là}

6 Jusqu'à peu, seuls certains professionnels connaissaient les " gardes »: terme par lequel on désigne le fait d'être constamment disponible, prêt à intervenir immédiatement en dehors des horaires habituels de travail, la nuit ou l'espace d'un week-end par exemple. Les inspecteurs de police, les pompiers, les médecins hospitaliers, bref les professionnels de l'urgence connaissent bien ces temps d'astreinte durant lesquels il ne faut pas qu'ils s'éloignent de leur téléphone ou qu'ils quittent leur alphapage. Mais la généralisation des radiotéléphones mobiles et des beepers, couplée à l'extension de l'urgence dans des branches de plus en plus nombreuses de l'activité économique (voir plus loin), est en train d'élargir l'astreinte à des catégories de salariés jusqu'alors restés en dehors de ce phénomène de gestion omniprésente de l'urgence.

7 Ainsi, Yves Lasfargue remarque que «depuis quelques mois, des ingénieurs informaticiens de grandes banques doivent être disponibles la nuit, le samedi et le dimanche, après avoir travaillé normalement le jour. Ils doivent porter en permanence un téléphone afin que l'on puisse les joindre en cas de panne sur les ordinateurs et les réseaux reliés aux distributeurs automatiques de billets. On constate que certains, peu habitués à l'astreinte, sont tellement stressés qu'ils décrochent le téléphone à 2 heures du 
matin, sans que celui-ci ait réellement sonné. Leur entreprise pense contrebalancer ce stress en leur versant une prime de 28,12 francs pour chaque intervention nocturne. $»^{2}$

Sous prétexte de sécurité, de concurrence ou d'urgence, des catégories entières de cadres sont gracieusement dotés par leur direction de radio-téléphones mobiles ou d'alphapages «personnels » qui permettent de les joindre en tout lieu et à toute heure. Si de nouvelles conventions collectives ne sont pas adoptées en la matière, le risque est grand d'assister à l'insidieuse colonisation de leur sphère privée par leur sphère professionnelle. Dans ce cas, on peut faire l'hypothèse très néo-corporatiste d'une future lutte entre catégories de cadres ayant pour enjeu la déconnexion professionnelle durant leurs occupations privées et de loisir, les gagnants étant ceux qui pourront se délester de l'urgence sur les perdants.

Les dirigeants d'entreprises et décideurs, toujours sur la brèche, s'auto-excluent évidemment de cette lutte qui n'opposerait que des cadres contraints par leur hiérarchie à effacer les frontières entre leur sphère professionnelle et leur sphère privée. Mais ils s'en auto-excluent désormais de deux façons bien distinctes: la première, classique pourrait-on dire, relève du fait que l'acteur dirigeant s'identifie totalement à son entreprise. Il n'y a pas lieu de faire de différence entre sa vie tout court (incluant donc sa vie privée) et sa vie professionnelle : elles ne font qu'un, il n'y a jamais déconnexion. La seconde, en voie de généralisation semble-t-il, est liée au développement des télécommunications : grâce à elles, un ou plusieurs cadres supérieurs « ont l'honneur » de fonctionner comme filtre entre les sollicitations de l'entreprise et du marché, et le dirigeant en week-end ou «momentanément injoignable». Lorsqu'on sait que, toujours grâce aux télécommunications, les employés de base ont de plus en plus tendance à s'en référer à la hiérarchie avant de prendre une initiative face à l'imprévu, on comprend mieux pourquoi un nombre croissant de cadres hyper-communicants "craquent " brusquement (burn ont) sans raison apparente...

\section{Les dangers de l'urgence généralisée}

10 En permettant des économies de temps et en donnant l'illusion d'un affranchissement des contraintes spatiales, l'ubiquité médiatique peut conduire au mythe quantitatif du bon choix. Ce mythe peut être résumé ainsi : le bon choix est celui qui est fait à partir du maximum d'informations concernant le sujet abordé. Autrement dit: plus le nombre d'informations est élevé, et plus le choix a des chances d'être bon. Application: les télécommunications permettant de recueillir un maximum d'informations, les choix s'optimisent. Cette vision peut conduire à deux écueils sur lesquels font naufrage bon nombre d'individus et d'organisations.

11 Le premier est de devoir faire face à une inflation d'informations dont la masse interdit un traitement efficace. Face à une pile de notes, une succesion de fax urgents et un sansfin de sollicitations téléphoniques, il faut aller vite. Dans cette accélération, synonyme de diminution du temps de réflexion, l'accessoire risque de recouvrir l'essentiel. Temps de réflexion, de mûrissement, mais aussi de retour sur le passé et de tension vers l'avenir, l'attente et le différé semblent ici chargés de négativité. L'inflation d'informations, l'extension de leurs champs et leur immédiateté produisent un environnement d'urgence généralisée. Non seulement il faut toujours être là, mais il faut aussi pouvoir répondre rapidement. La prise de distance nécessaire à la réflexion et à l'imaginaire court ainsi le risque d'être remplacée par une espèce de réflexe de zappeur allant au plus pressé. 

coup de fil a dès lors priorité sur la personne présente, le fax sur le courrier, et le beeper arrête tout, séance tenante. Comme si l'individu ou l'organisation se mettait aux ordre de l'urgence, l'advenant supplantant l'existant. Le danger réside dans l'extension généralisée de ce mode de réaction: il risque de se muer en véritable mode de fonctionnement. Focalisées sur la réaction aux sollications immédiates, personnes ou structures courent le risquent de perdre tout pouvoir stratégique au profit de pures tactiques d'adaptation à un environnement qu'elles ne maîtrisent plus. De réfléchi, volontaire et actif, le choix devient réactif, aléatoire et improvisé, et a donc toute chance d'être dépendant.

purieux est de constater que les personnes ayant adopté ce mode de fonctionnement sont souvent conscientes que ce qui est urgent sur le moment n'est pas nécessairement important à court terme, l'est rarement à moyen terme et presque jamais à long terme... Mais cette réflexion n'est énoncée que dans une situation d'interview et... ne les empêche pas de continuer à gérer prioritairement l'urgent. Cette attitude révèle la peur de rater quelque chose, de ne pas s'être trouvé où il fallait au bon moment, ou encore de n'avoir pas eu la bonne information à temps, symptômes de cette maladie encore mal définie de l'homme post-moderne que j'ai appelée le syndrome du zappeur³.

\section{L'hertziennisation du contrôle hiérarchique}

Pour les employés en déplacement ou géographiquement excentrés de leur direction, le fait d'être médiatiquement proche de leur hiérarchie est un facteur de sécurité : en cas de problème, il leur est possible de se décharger du poids d'une décision délicate (sur les pauvres cadres dont il a été question plus haut). Mais cette faculté est ambivalente : elle peut retrancher à la liberté ce qu'elle offre à la sécurité. Etant directement joignable, l'employé est en effet constamment sous contrôle. À tout moment, comme sur une chaîne de fabrication, la hiérarchie peut désormais vérifier son planning, sa rentabilité, son efficacité.

Les télécommunications permettent certes de supprimer un grand nombre des aléas perturbant l'efficacité des employés en déplacement. Mais ces aléas n'étaient pas toujours vécus négativement, loin s'en faut! Si le fait d'être en permanence en contact avec un centre de contrôle permet d'éviter les désagréments d'un bouchon routier ou ceux d'un rendez-vous manqué, il supprime aussi ces temps morts pour la rentabilité mais bien vivants pour l'employé. Temps d'un arrêt imprévu, d'une émotion visuelle, d'un regard ou d'un sourire échangé, temps de rêverie, de déconnexions éphémères, temps personnel, privé, intime, vécus dans l'espace du travail mais hétérogènes à son temps programmé. Pensés comme interférences, «bruits » ou désordre par l'économie de l'organisation, ces «temps perdus» sont supprimés ou balisés (arrêt café). Mais trop d'intégration et de programmation peuvent nuire et risquent de produire à la fois un déficit d'inédit et de surprise, et une espèce d'éloge du temps perdu, du temps gaspillé, de son utilisation irrationnelle: "J'ai l'impression que toutes ces techniques de communication, leur abondance même, créent un déterminisme (...) qui nous enlève certaines illusions. On en vient à souhaiter qu'il y ait une part d'incertitude et donc une possibilité que quelque chose qu'on ignore encore se produise.» "On a besoin de sécurité et à la fois d'improvisation, ce qui semble un peu contradictoire. Inconsciemment, je guette le grain de sable qui va enrayer la machine et nous obliger à l'improvisation. $»^{4}$ 
16 À trop vouloir fonctionnaliser et rentabiliser les employés en déplacement, on court par ailleurs le risque de les voir se désinvestir de leur responsabilité. Ainsi, un chauffeur routier ou un VRP peuvent voir leur planning modifié en pleine course pour urgence absolue. Le donneur d'ordre mesure sans doute mal le degré de désappointement qu'un tel changement peut produire. De planificateurs d'un temps imparti, ce chauffeur ou ce VRP deviennent fonctions programmables à distance. Leur capacité d'improvisation et la fierté même de leur métier s'en trouvent sans doute atteintes.

\section{L'instrumentalisation de la communication}

17 Le terme générique de télécommunications cache en fait deux grands types de communications. Dans le premier, l'information transmise condense à elle seule le motif de l'appel. Il s'agit la plupart du temps d'échanger de pures données instrumentales et fonctionnelles (une commande, un plan, une précision technique). Dans ce type de communication, le lien unissant les interlocuteurs relève d'une interdépendance fonctionnelle et formalisée. Le correspondant est appelé à cause de son statut et celui-ci suffit à définir la nature de l'échange. Il s'agit là de communications qu'Habermas qualifierait d'utilitaristes et stratégiques, c'est-à-dire uniquement orientées vers le succès de procédures techniques ou par des actions dont le but est d'adapter les moyens jugés les plus rationnels et efficients à des fins déterminées ${ }^{5}$. Le recours à tel ou tel moyen technique de transmission se fait alors sans état d'âme, pourvu que ce moyen soit le mieux adapté à la nature des informations échangées. Lorsqu'il est médiatisé, ce type de communication formalisée renvoie à proprement parler à la téléinformation.

18 Il en va bien différemment lorsque la communication est entendue non plus seulement comme simple échange d'informations mais aussi comme relation renvoyant à une communauté intersubjective. Ici, la communication vise tout autant (et souvent plus) à instituer, confirmer ou réactualiser par l'échange la nature de la relation interpersonnelle liant les interlocuteurs qu'à transmettre de purs constats, données ou énoncés fonctionnels. Le vécu intersubjectif de l'échange est central. Au-delà même de ce qui est dit, c'est ce qui est donné à comprendre de la relation qui importe. Le face à face est la situation idéale pour ce type de communication. Le terme de télécommunication devrait être réservé à ce second type de communication électroniquement médiatisée.

Sur la base de cette distinction, une nouvelle différenciation risque d'apparaitre au sein de l'entreprise: d'un côté "les riches» qui pourront nouer des rapports autres qu'instrumentaux avec leurs interlocuteurs, et « les pauvres » qui n'auront accès qu'à des interlocuteurs-fonctions, des interlocuteurs-statuts, en définitive des interlocuteurs-nonsujets avec lesquels le hasard, l'affect, la séduction seront évacués au profit d'une pure instrumentalisation de la communication. D'un côté la communication comme enrichissement potentiel, de l'autre sa chosification.

Or, le développement des outils de télécommunication semble augmenter la masse des communications du premier type (téléinformations) plutôt que celle du second type (communications intersubjectives). Le remplacement d'un face à face par un appel téléphonique n'est pas neutre, il y a immanquablement une forte déperdition d'informations intersubjectives. Cela ne veut pas dire que l'intersubjectivité ne passe pas par téléphone, mais que, globalement, surtout lorsque les appels sont professionnels, il y en a moins: il faut la plupart du temps aller vite, à l'essentiel, c'est-à-dire à la 
transmission efficace d'informations pragmatiques. De la même façon, le remplacement d'un courrier par un fax se traduit par une certaine désinvolture formelle, seule les données transmises et surtout leur urgence importent, les messages d'accompagnement sont réduits à leur plus simple expression. En quelque sorte, l'urgence permet la brusquerie.

Or les victimes de cette brusquerie sont elles-mêmes productrices de communications formalisées laissant peu de place à la chaleur humaine, et même de moins en moins aux formes classiques de politesse. Il en résulte une tension que j'ai pu constater de façon récurrente chez de grands télécommunicants entre une rationalisation instrumentale croissante de leur vie (efficacité) et une sorte de déficit en sentiments (affect) révélée par cette trop grande ou trop unique rationalisation. D'un côté la traduction stratégique de l'agir communicationnel («il faut que ça rapporte quelque chose»), et de l'autre l'aspiration confuse à «autre chose ", à un rapport moins instrumental à la réalité : les personnes et les institutions doivent pouvoir être pensés autrement que sur le mode de relations à de simples objets, ressources ou fonctions. Cette tension se solde presque toujours par des tiraillements, une inquiétude, un malaise et parfois même de la souffrance.

\section{D'expériences individuelles éclatées à une réflexion collective sur les usages des télécommunications en entreprise}

Pour l'instant, la majorité des effets pervers des télécommunications sont vécus individuellement. La personne de garde appelée en pleine nuit est isolée (de fait, l'hyper branché est presque toujours isolé : s'il ne l'était pas, il ne serait pas branché). Mais, la même nuit, ce seront des centaines ou peut-être des milliers de personnes qui seront réveillées par un appel professionnel: si l'expérience est individuelle, le problème est collectif. De la même façon des milliers de personnes font l'expérience d'un surcroît d'urgence, flirtent quotidiennement avec les limites du dernier moment, s'installent malgré eux dans un stress permanent.

Le coût social du stress, de l'énervement ou du malaise provoqués par les aspects négatifs qui ont été évoqués n'est pas mesurable. Individuellement vécus, ils semblent renvoyer à des problèmes d'ordre psychologique. Pourtant les problèmes qu'ils posent sont bien sociaux et culturels. Il est donc temps d'engager une réflexion et un débat collectif sur ces thèmes. Un nouveau droit doit être défendu : le droit à la déconnexion et à l'isolement, le droit de refuser de porter un bayerjour et nuit ou d'être radiotéléphoniquement partout joignable. De nouvelles règles de politesse doivent être imaginées qui respectent le droit qui vient d'être énoncé, mais aussi la dignité des personnes qui ne sauraient être réduites à être des fonctions ou des ressources. 


\section{NOTES}

1. L'ensemble des arguments qui suivent sont issus des conclusions d'une série de recherches menées ces cinq dernières années pour France Télécom auprès de gros utilisateurs de terminaux de télécommunication (visiophone, téléphone, radio-téléphone, alphapage et fax). La méthodologie employée a été celle de l'observation participante, d'entretiens individuels et de l'intervention sociologique.

2. "Le harcèlement des télécommunications ». Le Monde initiatives, 7 octobre 1992,

3. Voir Francis Jauréguiberry, Un goût d'ubiquité, CNRS-Université de Pau, 1989.

4. Témoignages, in Francis Jauréguiberry, Un goût d'ubiquité, CNRS-Université de Pau, WW, p. 57.

5. Jürgen Habermas, Théorie de l'agir communicationnel, trad. française, Paris, Fayard, 1987, tome 1, pp 295-296

\section{RÉSUMÉS}

Que ce soit au niveau de l'adaptabilité au marché, de l'optimisation des fonctions ou de l'organisation du système de l'entreprise-réseaux, les télécommunications deviennent chaque jour plus indispensables aux entreprises modernes. Mais les économies de temps et de déplacements qu'elles permettent ne doivent pas faire oublier les bouleversements qu'elles peuvent engendrer dans les rapports humains. Ces bouleversements sont loins d'être tous positifs: quelques uns sont au contraire négatifs sous certains aspects que l'auteur qualifie d'effets pervers : le fait d'être joignable à tout heure et en tout lieu qui peut se traduire par une « colonisation » de la sphère privée par la sphère professionnelle, la généralisation de l'urgence, le manque de distance et de réflexion que cela peut susciter, l'herziennisation du contrôle hiérarchique et l'instrumentalisation des rapports humains. Autant d'aspects qui risquent d'engendrer de nouveaux rapports conflictuels autour d'enjeux inédits.

Whether it is a question of market adaptibility, optimisation of its Junctions or the organisation of the business network system, telecommunications are becoming more and more indispensable to the modern company. However, time and energy saving which are the result should not hide the upheavals which can be caused in human relationships. These upsets are not all positive. Some be quite negative, in conditions which the author qualifies as perverse The fact that on can be contacted anywhere at any time finishes as a kind of "colonisation" of private life by professional life. Everything becomes urgent, there is less time and distance to give perspective in decision making, hierarchical control becomes technological and human relations become instrumentalised These are just some of the factors which risk creating conflictual relations around as yet unknown stakes. 
AUTEUR

FRANCIS JAURÉGUIBERRY

Francis Jauréguiberry est responsable de recherche à l'URA 911 du CNRS et maitre de conférences de sociologie à l'Université de Pau. 